\title{
Towards Probabilistic Estimation of Quality of Online Services
}

\author{
Le-Hung Vu and Karl Aberer \\ Swiss Federal Institute of Technology Lausanne (EPFL), \\ School of Computer and Communication Sciences, \\ CH-1015 Lausanne, Switzerland \\ \{lehung.vu|karl.aberer\}@epfl.ch
}

\begin{abstract}
Accurate estimation of quality of online services is both an important and difficult problem, since a service has many interdependent quality attributes influenced by several contextual factors. It is even more challenging as quality ratings come from sources with unknown reliability, each source may rate a service on different quality aspects. Although several solutions have been proposed, there is little work addressing all these issues thoroughly. In this paper, we show that domain knowledge on service structure and related constraints, such as causal dependencies among quality attributes and contextual factors, while widely available, can be exploited to effectively address the above issues in a theoretically-sound framework. Theoretical analysis shows that computational cost of the approach is acceptable, and accurate evaluation of service quality requires a reasonable number of user feedback, provided services have a small number of quality attributes and contextual factors.
\end{abstract}

\section{Introduction}

More and more traditional services are being advertised, discovered and requested electronically via the Internet. Advertising sites such as Craigslist ${ }^{1}$ have become widely popular with numerous publications of services across different domains: from data-hosting, travel planning to house relocation or car rental services.

Accurate estimation of quality of such online services, especially in competitive business scenarios, is of paramount importance. Quality rating is one of the decisive criteria influencing a user in selecting a service among several functionally equivalent ones. For instance, between two online travel planning services, a user would aim for the one with better route coverage, more user-friendly, and offer plans with lower price and transit time.

Ratings on quality of online services can be obtained in many ways. Automatic monitoring and measuring ser-

\footnotetext{
${ }^{1}$ http://www.craigslist.org
}

vice quality are proposed in, for example, $[2,16]$. Such approaches are appropriate to measure network-related quality metrics of a Web service, e.g., service response-time or availability, but inapplicable to other domain-dependent quality properties of an online service in general, e.g., whether the service conforms to its advertised functions or whether it is easy to use the service. Moreover, in practice users are even more interested in these domain-dependent quality features. An extensive review of such quality attributes across service domains is given in [1].

Alternatively, feedback from previous users can be used as a relevant source to rate most quality attributes of a service $^{2}$. Feedback is available in various places, from extended registries [3] to professional forums and social networks. In this case, a fundamental issue is the reliability or trustworthiness of feedback sources, as reports from unknown people can either be reliable or biased depending on the innate behaviors and motivation of the ones sharing the feedback. For example, competing service providers have strong incentives to advertise their services as having the highest possible quality and to badmouth quality of their competitors in order to increase their revenues $[4,5]$.

Accurate estimation of service quality is even more challenging since quality signals are multi-dimensional, contextsensitive, and may be nondeterministic. A service has several interdependent quality attributes whose values may be influenced by various contextual factors nondeterministically. Examples are dependencies between membership type of a data hosting service, e.g., Amazon S3, and download/upload bandwidth available to a member given his network connection speed. These issues should be considered adequately when devising an appropriate solution for estimating quality of a service from feedback data.

Several approaches propose to use user feedback to evaluate quality ratings, namely [7, 19-21]. [19] selects a service based on a number of independent QoS attributes, and suggests to identify requesters to avoid report flood-

\footnotetext{
${ }^{2}$ Since we evaluate quality of online services in general, henceforth the term service refers to an online service, not necessarily a Web service
} 
ing. [20] considers relationships among quality attributes, yet it assumes feedback sources are reliable. [7] evaluates services based on difference among their QoS vectors. [6] uses a collaborative filtering approach: estimate quality of a new service by using feedback of users with similar experience. [21] proposes to select only popular services by analyzing a network of related services. In summary, existing approaches mostly consider independent quality attributes of a service, and some assumes full reliability of the ratings. Also, many solutions are based on ad hoc heuristics that are not theoretically sound and the notion of context are usually not included. Only [22] considers the case where a user may give feedback on a few quality aspects of the service, resulting in a sparse set of quality ratings. There is little work that adequately addresses the multi-dimensionality, contextsensitivity, nondeterminism of service quality signals, as well as the reliability of contributing feedback sources.

This paper presents an overall framework to use service domain knowledge to model and estimate ratings on quality of services probabilistically, taking into account all these above issues. The key idea is that structure of a service and related constraints, such as causal dependencies among quality attributes, contextual factors, are generally available in a service domain. We exploit this information for the modeling and learning of service quality in many aspects. First, we model quality capabilities of a service as a fixedstructure Bayesian network to facilitate subsequent probabilistic learning and inferences on it. Second, we use constraints among quality attributes, contextual factors, possible malicious behaviors of raters, and their reported values to define conditional probabilities of the model and to filter out invalid ratings, which helps to improve performance of the service quality estimation. Third, prior beliefs on feedback sources' reliability and service provider's behavior can be used as additional input to train the model. Our proposed framework has many advantages:

- it offers a theoretically-sound solution to effectively address the issues of multi-dimensionality, contextsensitivity, and reliability of service quality ratings, while applicable to many service domains.

- it enables users to subjectively model and evaluate various quality dimensions of interested services according to personalized preferences, prior beliefs and available information on trustworthiness of the feedback sources.

- given the service model, the estimation of service quality works well with many missing values in collect reports. After the model has been trained with available reports, it is possible to use the model to estimate quality level of unobserved quality factors from ratings on some other quality features of the service. Furthermore, correlations between opinions of honest and trusted users are naturally integrated in the training step and help to identify reliable reports and isolate biased ones, thus increase the evaluation accuracy. This is demonstrated in our previous work [8]: the estimation error is low even with many missing values from reports of several biased sources.

To the best of our knowledge, this work is the first one that effectively exploits available domain knowledge for accurately evaluation of service quality. Furthermore, while we focus on modeling and assessing service quality in the paper, the methodology is applicable to other service's nonfunctional properties with probabilistic dependencies.

\section{System model and notations}

\section{Framework overview}

Fig. 1 gives an overview of our approach to probabilistic modeling and estimation quality of a service from ratings by many feedback sources. The modeling step builds a QoS generative model of a service to represent its quality capabilities. The learning (training) step trains the model with feedback on quality of the service from many sources to learn its unknown parameter. The third step estimates the service quality level under a certain context by probabilistic inference on the model learnt from two previous steps.

This framework can be integrated into a service reputation management framework [9] or a service search engine, namely Seekda ${ }^{3}$ to help users selecting the best services. In this paper we will introduce candidate algorithms for the above steps to evaluate quality of an example service and analyze in details computational complexity of these algorithms to demonstrate the possibility of our approach. These algorithms, however, are subject to many optimizations and can be replaced by better ones, as discussed later on.

Denote $\mathcal{Q}$ the set of quality parameters of a service, where each $q \in \mathcal{Q}$ is assumed to have discrete values. While this assumption largely simplifies our problem and subsequent analysis, it is both realistic and advantageous. First, many quality attributes either have categorical values or are best represented with ranges of values due to their uncertain nature. Second, a rating on service quality is in fact the conformance between the quality values promised and delivered by the provider, as evaluated by the user. Such ratings are best modeled as discrete grading scales, as normally used in rating hotels or travel planning services. Similarly, let $\mathcal{E}$ be the set of contextual factors affecting values of quality parameters in $\mathcal{Q}$. In this paper we only consider the case where contextual factors have discrete/categorical values. The same methodology, however, applies in the case of continuous contextual factors and quality signals.

Motivating example: Consider a data hosting service similar to Amazon S3 or services by other data hosting providers. The following quality attributes are of our interests: its maximal number of concurrent downloads $M$, its download speed $D$ and its upload speed $U$. Values of

\footnotetext{
${ }^{3}$ http://seekda.com/
} 


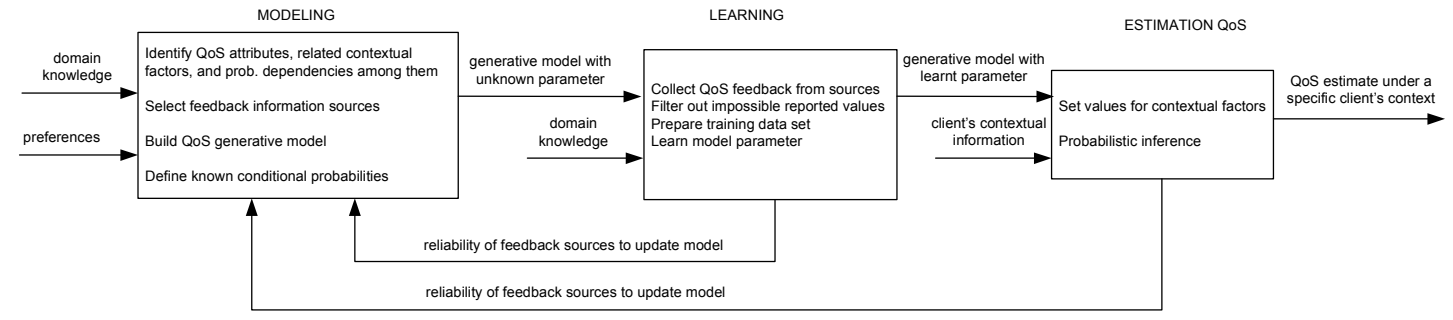

Figure 1: The framework overview.

these attributes are decided by the subscription price $P$ and the Internet connection type $I$ of the service consumer. In other words, $P$ and $I$ define the context, or the client-side setting in which the provider promises to offer its data hosting service with specific quality level of $M, D$, and $U$.

Fig. 2(a) shows a Bayesian network-based model of quality of the data hosting service with state spaces of all nodes and probabilistic dependencies among them. Such information is well-known in the data hosting domain and may even be declared by the provider in his service advertisement. Fig. 2(a), with conditional probabilities of each variable given state of parent nodes, is example of a $Q o S$ generative model of the data hosting service.

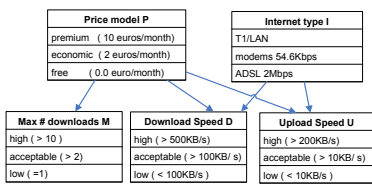

(a)

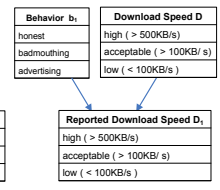

(b)

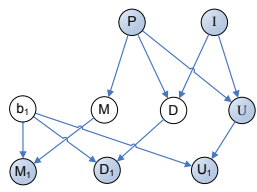

(c)
Figure 2: (a) Example quality attributes and related contextual factors of a data hosting service, state spaces of each node, and dependencies among them; (b) Dependencies among the trustworthiness (reporting behavior) of an untrusted feedback source and its corresponding reported values; (c) The QoS generative model of the data hosting service in simple form.

A user may obtain reports (ratings) on quality of a service from a list of sources $\mathcal{I}$, only some of which are reliable. Reliable (trusted) sources $\mathcal{T} \subseteq \mathcal{I}$ may include, for example, third-party/commercial QoS monitoring services. Untrusted sources $\mathcal{I} \backslash \mathcal{T}$ are usually previous service users. The trusted sources and those quality attributes to be monitored by them shall be private information of the learning user. Otherwise, an adversary may disguise as an honest reporter to manipulate the QoS prediction effectively. For example, the adversary can provide reliable information only on those quality attributes monitored by trusted sources, while reporting biased feedback on the others.

Let $\mathcal{U}$ be the set of variables in a QoS generative model of a service. A node $x \in \mathcal{U}$ represents either a quality attribute, a contextual factor, the trustworthiness of an information source, or the rating on a quality attribute. The domain value of $x$, or its state space, is denoted $\mathcal{D}_{x}$. A directed edge from one node to another denotes a probabilistic or causal dependency between two variables. For brevity, we denote $\pi_{x}$ the set of parent nodes of $x$. If $x$ represents a quality attribute in $\mathcal{Q}$, the set of contextual factors in $\mathcal{E}$ that $x$ directly depends on is $\phi_{x}=\pi_{x} \cap \mathcal{E}$.

The realization of $x$ to some value $\mathrm{v} \in \mathcal{D}_{x}$ is denoted as $x=\mathrm{v}(x \neq \mathrm{v}$ is defined similarly). Whenever it is irrelevant to mention $\mathrm{v}$, we use the brief notation $x^{*}$. Similarly, for any given set $\mathcal{S}$ of random variables, we denote $\mathcal{S}^{*}$ the joint event in which each variable $s \in \mathcal{S}$ is assigned certain value in $\mathcal{D}_{s}$. The conditional probability that $x$ gets some value given states of its parents $\pi_{x}$, or a conditional probability table $(C P T)$ entry of the node $x$, is simply $\operatorname{Pr}\left(x^{*} \mid \pi_{x}^{*}\right)$.

We then introduce the following important concepts.

Definition 1. The QoS generative model of a service is a Bayesian network $\langle\mathcal{U}, \mathcal{D}, \theta\rangle$ with node set $\mathcal{U}$, edge set $\mathcal{D}$, and parameter $\theta$. The tuple $\langle\mathcal{U}, \mathcal{D}\rangle$ is a directed acyclic graph where $\mathcal{D}=\left\{\langle p, x\rangle, x \in \mathcal{U}, p \in \pi_{x}\right\}$ defines probabilistic dependencies among nodes in $\mathcal{U}$. The parameter $\theta$ is the set of unknown conditional probability table $(C P T)$ entries $\operatorname{Pr}\left(x^{*} \mid \pi_{x}^{*}\right)$ of each node $x \in \mathcal{U}$.

Definition 2. A $Q o S$ rating by a source $j \in \mathcal{I}$ at time $t$ on a service is defined as $\left\langle j, t, \mathcal{V}_{j}^{*}\right\rangle$, where $\mathcal{V}_{j}^{*}$ denotes a set of reported values of $j$ on a subset $\mathcal{V}_{j} \subseteq \mathcal{Q} \cup \mathcal{E}$. Specifically, $\mathcal{V}_{j}^{*}=\left\{x=\mathrm{r}_{\mathrm{jt}}(x) \mid x \in \mathcal{V}_{j}\right\}$, where $\mathrm{r}_{\mathrm{jt}}(x) \in \mathcal{D}_{x}$ is the reported value of $j$ on $x$ at time $t$.

In general a source $j$ may report only on a number of quality attributes or contextual factors $\mathcal{V}_{j}$. Thus values of several quality attributes and contextual factors may be missing in a QoS rating. To estimate the unknown parameter $\theta$ of a QoS generative model, related QoS ratings from many sources are combined to build a training set, which includes many observations (samples) on the model (Def. 3).

Definition 3. An observation or a sample on a QoS generative model is defined as $\mathbf{v}_{\mu}=\left\{x=\mathrm{x}^{\mu}, x \in \mathcal{O}\right\}$, where $\mathrm{x}^{\mu} \in \mathcal{D}_{x}$ is a rating value on a node $x \in \mathcal{O}$, and the set $\mathcal{O} \subseteq \mathcal{Q} \cup \mathcal{E}$ is a subset of quality attributes or contextual factors whose values during a measurement epoch $\mu$ can be obtained by combining ratings from some sources.

\section{Service quality modeling}

Given own knowledge on the reliability of feedback sources, a user may set up a QoS generative model of the 
following types. The basic QoS generative model (Fig. 3a) is applicable when the user collects ratings from own experience and only trusted sources. The extended QoS generative model (Fig. 3b) is used when the user collects feedback from many sources $\mathcal{I}$, only a subset $\mathcal{T}$ of which is reliable. A QoS generative model is built with the following steps:

1. Identify relevant quality attributes $\mathcal{Q}$ of the service, related contextual factors $\mathcal{E}$ and their domain values. Discretize values of a continuous quality attribute into ranges if necessary. Identify the causal/probabilistic relationships among $\mathcal{Q}$ and $\mathcal{E}$. This results in a basic QoS generative model as shown in Fig. 3(a).

2. For each untrusted feedback source $j \in \mathcal{I} \backslash \mathcal{T}$, add a node $b_{j}$ to represent the trustworthiness of $j$. For each $b_{j}, j \in$ $\mathcal{I}$ and for each $q_{i} \in \mathcal{Q}$, add a node $v_{i j}$ with the same domain as $q_{i}$. Add a directed link from each $b_{j}$ and from each $q_{i}$ to $v_{i j}$. Thus, $v_{i j}$ represents the rating of a source $j \in \mathcal{I}$ on a quality attribute $q_{i} \in \mathcal{Q}$. The following nodes are marked observable: all nodes $v_{i j}, \mathcal{E}$, and each $q_{s}$ rated by a trusted source $s \in \mathcal{T}$. Remaining nodes are hidden nodes. This step results in an extended QoS generative model (Fig. 3b) with an unknown parameter $\theta$.

3. Well-known constraints among values of nodes are exploited to define certain CPT entries of the model. Particularly, one may set $\operatorname{Pr}\left(v_{i j}=\mathrm{v} \mid b_{i}=\right.$ reliable, $q_{j}=$ $\mathrm{v})=1$, and $\operatorname{Pr}\left(v_{i j}=\mathrm{v} \mid b_{i} \neq\right.$ reliable, $\left.q_{j}=\mathrm{v}\right)=0$ for any $q_{i} \in \mathcal{Q}, \mathrm{v} \in \mathcal{D}_{q_{i}}, j \in \mathcal{I} \backslash \mathcal{T}$. Other value constraints of quality and contextual attributes can be exploited to set CPT entries of related nodes. Formally, a constraint may be of the form $\bigwedge_{x \in \mathcal{X}} x^{*} \rightarrow y^{*}$ or $\bigwedge_{x \in \mathcal{X}} x^{*} \rightarrow(y \neq \mathrm{v})$, for some $\mathcal{X} \subseteq \mathcal{U}$, some $y \in \mathcal{U}$, and some $\mathrm{v} \in \mathcal{D}_{y}$. Define $\mathcal{K B}$ be the domain knowledge built from those constraints. For each $x \in \mathcal{U}$, the following rules apply: if $\mathcal{K B} \models\left\{\pi_{x}^{*} \rightarrow x^{*}\right\}$, we define $\operatorname{Pr}\left(x^{*} \mid \pi_{x}^{*}\right)=1^{4}$. If $\mathcal{K B} \models\left\{\pi_{x}^{*} \rightarrow(x \neq \mathrm{v})\right\}$, set $\operatorname{Pr}\left(x=\mathrm{v} \mid \pi_{x}^{*}\right)=0$. Prolog programs can help to analyze such complex constraints to set values of related CPT entries.

The above steps result in a (personalized) QoS generative model of the service, with some pre-defined CPT entries. Normally, a generative model is set up once for each service type in a domain, with possible help of domain experts. We emphasize that it is possible to include dependencies among quality attributes $q_{i}$, or among contextual factors $e_{l}$ in Fig. 3(a,b). These dependencies are not shown for the presentation clarity. However, our proposed algorithms in coming sections are generic enough to handle these cases. Also, we assume that values of contextual factors are verifiable and thus are observable variables. This is not a strong assumption since there is no direct incentive for feedback sources to manipulate such values, but only values of concerned quality parameters. Nevertheless, whenever contex-

\footnotetext{
${ }^{4}$ We use the notation $\models$ to represent the deduction (provability) of a fact from a knowledge-base in a Hilbert-style deduction system
}

tual values are subject to manipulation, it is trivial to extend the above models and use the same approach with contextual factors marked as hidden nodes. It is also noteworthy that available domain constraints are very useful as they help to define many conditional probabilities of the model (step 3.) and thus reduce the size of the parameter set $\theta$ to be estimated in later steps.

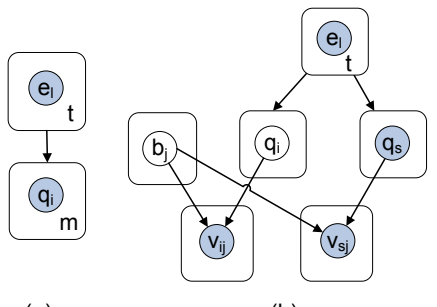

(a)

(b)

Figure 3: (a) The basic QoS generative model of a service; (b) The extended QoS generative model. A rounded square surrounding a node represents a group of similar variables, possibly with the number of variables in the group. Shaded nodes are observable (visible) variables whose values are known or obtained in some ratings, whereas blank nodes are hidden variables whose values are completely unknown.

Modeling example: Consider the basic QoS generative model for the previous data hosting service (Fig. 2a). Suppose that feedback on $U$ is provided by a trusted source, and feedback on $M, D$, and $U$ is obtained from an untrusted source with reporting behavior $b_{1}$. We use $M_{1}, D_{1}$, and $U_{1}$ to denote reported values by the untrusted source on $M, D$, and $U$, respectively. $P, I, U, M_{1}, D_{1}$, and $U_{1}$ are marked observable. Fig. 2(b) shows possible dependencies between the trustworthiness $b_{1}$ of a source and reported values $U_{1}$ on the download speed of the data hosting service. A feedback source observing a certain quality level may either report the same value, or deliberately give higher or lower rating value. E.g., a user may give a bad rating on service of its competitors irrespective to the quality it actually perceives. These reporting behaviors are denoted as reliable, advertising, and badmouthing respectively, i.e., the feedback source is reliable with some unknown probability. Fig. 2(c) shows the QoS generative model after the second modeling step.

We then exploit the following constraints to define some CPT entries of the model. Given the above reporting behavior model, for any $\mathrm{u} \in \mathcal{D}_{U}$ we have $\operatorname{Pr}\left(U_{1}=\mathrm{u}\right.$ $b_{1}=$ reliable, $\left.U=\mathrm{u}\right)=1.0, \operatorname{Pr}\left(U_{1}=\right.$ high $\mid b_{1}=$ advertising, $U=\mathrm{u})=1.0$, and $\operatorname{Pr}\left(U_{1}=\right.$ low $\mid b_{1}=$ badmouthing, $U=\mathrm{u})=1.0$. Also, if the maximal number of downloads is deterministically defined by the price, we set $\operatorname{Pr}\left(M=\right.$ high $\mid P=$ premium, $\left.I^{*}\right)=1$, $\operatorname{Pr}\left(M=\right.$ acceptable $\mid P=$ economic, $\left.I^{*}\right)=1$, and $\operatorname{Pr}\left(M=\right.$ low $\mid P=$ free, $\left.I^{*}\right)=1$. Thus after the third modeling step we have an extended QoS generative model for the data hosting service with certain known CPT entries. 
The unknown parameter $\theta$ of the model consists of the remaining unknown CPT entries of $b_{1}, M$, and $D$.

Many existing works use certain instances of our above generative models for their QoS prediction, though the modeling is not stated explicitly. For example, those approaches considering a number of independent quality attributes [19] consider a generative model of independent nodes $q_{i}$. Those work assuming the total reliability of feedback sources $[6,20]$ simply use the basic QoS generative model and propose a training algorithm based on some heuristics to estimate the model parameter $\theta$. As a result, this work provides a framework to apply various probabilistic machine learning techniques to evaluate service quality which generalizes existing approaches.

\section{Training and estimating service quality}

Before using the QoS generative model for the estimation of service quality, we need to train the model to learn its unknown parameter $\theta$. Specifically, collected QoS ratings from many sources are used as training data to update and estimate remaining unknown CPT entries of the model. Training data preparation: A training data set includes many samples on the QoS generative model, each sample is obtained by combining related QoS ratings from different sources during a measurement epoch (Def. 3).

As in the modeling, the knowledge base $\mathcal{K B}$ built from domain constraints can also be used to filter out impossible observations in the report data set. For example, in the data hosting service domain, the maximum concurrent number of downloads for a subscribed user must be greater than 1 , meaning that $\operatorname{Pr}(M=$ low $\mid P \neq$ free $)=0$. Thus any report by a source $j$ of the form $\{M=$ low, $P=$ economic $\}$ or $\{M=$ low, $P=$ premium $\}$ is impossible and should be filtered out.

Consider a QoS rating $\left\langle j, t,\left\{x=\mathrm{r}_{\mathrm{jt}}(x) \mid x \in \mathcal{V}_{j}\right\}\right\rangle$ submitted by a source $j$ (see Def. 2). This report is removed if it violates certain domain constraints in $\mathcal{K B}$. Such a violation is formally equivalent to:

$$
\exists x: \mathcal{K B}, \bigcap_{y \in V_{j}, y \neq x}\left\{y=\mathrm{r}_{\mathrm{jt}}(y)\right\} \models\left\{x \neq \mathrm{r}_{\mathrm{jt}}(x)\right\}
$$

Violations as (1) can be detected by Prolog programs to eliminate invalid ratings of a source $j$. As $j$ is likely to be unreliable, its related nodes in Fig. 3b. $\left(b_{j}, v_{i j}: q_{i} \in \mathcal{Q}\right)$ are also eliminated. This preprocessing reduces the learning cost in many ways: it reduces the size of the training data set, while preserving most relevant information for the learning phase. Also, many nodes related to unreliable sources are eliminated, thus simplifying the model and reducing the number of parameters to be estimated.

Ratings on quality of the target service are transformed into observations on the QoS generative model as in Algorithm 1. The function selectRatings $\operatorname{In} \operatorname{Epoch}(\mu, \mathcal{R})$ returns those QoS ratings in the set $\mathcal{R}$ whose timestamps $t$ are within a measurement epoch $\mu$. The function findReportedNode $(j, x)$ returns the node $v$ that represents the reported value by $j$ on the node $x$.

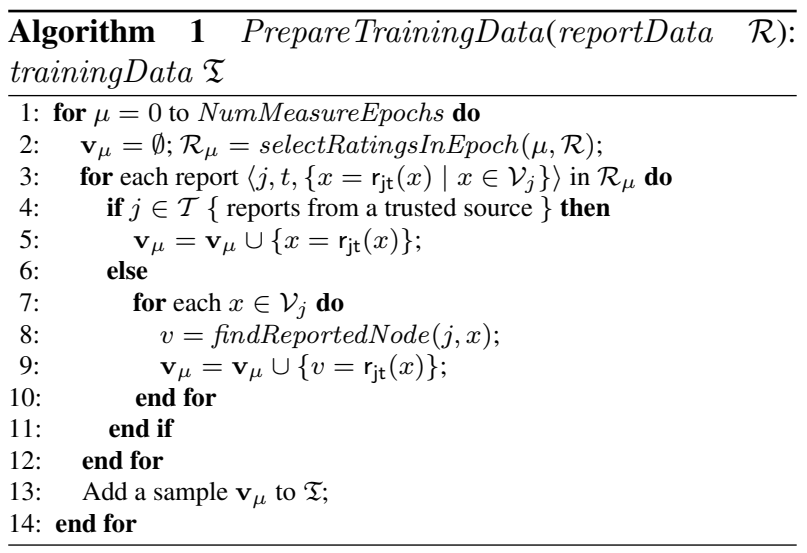

Learning by Maximum Likelihood Estimation (MLE): The unknown parameter of a QoS generative model can be estimated with traditional MLE techniques. This applies when all nodes of the model are observable, e.g., the case of a basic QoS generative model, or if training samples do not contain missing values. Consider a simpler version of the example data hosting service with two QoS attributes $D$ and $U$ as shown in Fig. 4. Assume that feedback on $D$ and $U$ is collected from an untrusted source with behavior $b_{1}$, and feedback on $U$ is also provided by a trusted source.

As explained before in section 3, for any value $\mathrm{d}$ of $D$, $\operatorname{Pr}\left(D_{1}=\mathrm{d} \mid b_{1}=\right.$ reliable, $\left.D=\mathrm{d}\right)=1, \operatorname{Pr}\left(D_{1}=\right.$ high $\mid$ $b_{1}=$ advertising, $\left.D=\mathrm{d}\right)=1, \operatorname{Pr}\left(D_{1}=\right.$ low $\mid b_{1}=$ badmouthing, $D=\mathrm{d})=1$. Similarly, for any $\mathrm{u} \in \mathcal{D}_{U}$ : $\operatorname{Pr}\left(U_{1}=\mathrm{u} \mid b_{1}=\right.$ reliable, $\left.U=\mathrm{u}\right)=1, \operatorname{Pr}\left(U_{1}=\right.$ high $\mid b_{1}=$ advertising, $\left.U=\mathrm{u}\right)=1, \operatorname{Pr}\left(U_{1}=\right.$ low $\mid$ $b_{1}=$ badmouthing, $\left.U=\mathrm{u}\right)=1$. Unknown CPT entries of the model in Fig. 4 are: $\operatorname{Pr}\left(b_{1}=\right.$ reliable $)=h, \operatorname{Pr}\left(b_{1}=\right.$ advertising $)=a, \operatorname{Pr}(D=$ high $)=x, \operatorname{Pr}(D=$ low $)=m$, and $\operatorname{Pr}(U=$ high $)=p, \operatorname{Pr}(U=$ low $)=q$. The unknown parameter of the model is thus $\theta=\{h, a, x, m, p, q\}$.
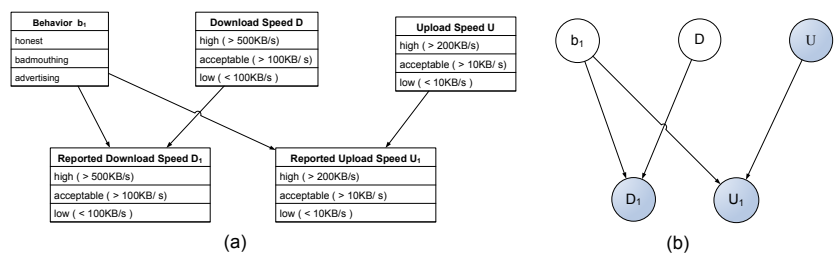

Figure 4: The generative QoS model of a data hosting service with two QoS parameters $D$ and $U$, monitored by two sources, one of which is untrusted with behavior $b_{1}$.

Suppose that we have a training data set $\mathfrak{T}=\left\{\mathbf{v}_{\mu}, 1 \leqslant\right.$ $\mu \leqslant N\}$ from feedback on $D, U$ built according to Algorithm 1. Each observation $\mathbf{v}_{\mu}$ is the combination of ratings 
from the trusted source (on $U$ ) and the untrusted source (on $D$ and $U)$, so $\mathbf{v}_{\mu}=\left\{U=\mathrm{u}^{\mu}, U_{1}=\mathrm{u}_{1}^{\mu}, D_{1}=\mathrm{d}_{1}^{\mu}\right\}$. The probability of getting an observation $\mathbf{v}_{\mu}$ is:

$$
\begin{aligned}
\operatorname{Pr}\left(\mathbf{v}_{\mu} \mid \theta\right) & =\operatorname{Pr}\left(u^{\mu}, u_{1}^{\mu}, d_{1}^{\mu} \mid \theta\right) \\
& =\sum_{b_{1}, D} \operatorname{Pr}\left(b_{1}\right) \operatorname{Pr}(D) \operatorname{Pr}\left(u^{\mu}\right) \operatorname{Pr}\left(d_{1}^{\mu} \mid b_{1}, D\right) \operatorname{Pr}\left(u_{1}^{\mu} \mid b_{1}, U\right)
\end{aligned}
$$

We select $\theta$ maximizing the $\log$ likelihood $L L(\theta)$ of obtaining the training set $\mathfrak{T}$, i.e., $\theta=\operatorname{argmax}_{\theta} L L(\theta)=$ $\sum_{\mu} \operatorname{Pr}\left(\mathbf{v}_{\mu} \mid \theta\right)$. Basic transformations give $\mathrm{us}^{5}$ :

$$
\begin{aligned}
L L(\theta)= & \sum_{\mu} \log \operatorname{Pr}\left(u^{\mu}\right)+\sum_{\mu} \log \left\{1_{\left\{u_{1}=u_{1}^{\mu}\right\}} \operatorname{Pr}\left(d_{1}^{\mu}\right) h+\right. \\
& \left.1_{\left\{u_{1}^{\mu}=d_{1}^{\mu}=\text { high }\right\}} a+1_{\left\{u_{1}^{\mu}=d_{1}^{\mu}=\text { low }\right\}}(1-a-h)\right\}
\end{aligned}
$$

One may readily estimate $\theta$ from reported values $\mathrm{u}^{\mu}, \mathrm{u}_{1}^{\mu}, \mathrm{d}_{1}^{\mu}$ from (2). E.g., we have $p=\frac{\sum_{\mu} 1_{\left\{\mathrm{u}^{\mu}=\text { high }\right\}}}{\sum_{\mu} 1}$ and $q=\frac{\sum_{\mu} 1_{\left\{u^{\mu}=\text { low }\right\}}}{\sum_{\mu} 1}$, similar to standard majority voting techniques for ratings on $U$. The reliability of the feedback source $(h)$ can be estimated numerically via standard optimization techniques. This results in an estimate of $h$ based on similarity between ratings $u^{\mu}$ of the unknown source and ratings $u_{1}^{\mu}$ of the trusted one, similar to intuition.

Learning by EM algorithm: For real services with many quality attributes and contextual variables, the resulting QoS generative models are more complex. Additionally, the training data set is likely to contain many missing values since each source may report on only a number of quality attributes. Direction maximization of the log likelihood function $L L(\theta)$ as above becomes non-trivial and does not give a closed-form solution of the estimated parameter $\theta$.

Many approaches for estimating the parameter $\theta$ under such situations are applicable [11]. In this paper, we propose the Expectation-Maximization (EM) algorithm [12] to estimate $\theta$ for its many advantages. First, it works well on any QoS generative model and is especially useful if the $\log$ likelihood function is too complex to be directly optimized. Furthermore, it deals with incomplete data and converges rapidly. Its main disadvantages are the possibility to reach to a sub-optimal estimate (a local maximum of the log likelihood) with a bad initialization. However, we believe that it is possible to have an acceptably good initialization of $\theta$ to compensate these disadvantages, given personalized beliefs of the learning user on the behaviors of service providers and raters. The EM algorithm is given in Algorithm 2.

Line 1 of Algorithm 2 initializes unknown CPT entries of the generative model. Each conditional probability $\operatorname{Pr}\left(x^{*} \mid \pi_{x}^{*}\right)$ can be initialized in many ways depending on available information: from prior beliefs of the user, as in the service advertisement by the provider, or as completely random. For the data hosting service, the learning user may assign $\operatorname{Pr}\left(b_{j}\right)$ with his or her subjective belief on the trustworthiness of the source $j$. CPT entries of $M, D, U$ in the

\footnotetext{
${ }^{5}$ The function $1_{A}$ evaluates to 1 if $\mathrm{A}$ is true and 0 otherwise
}

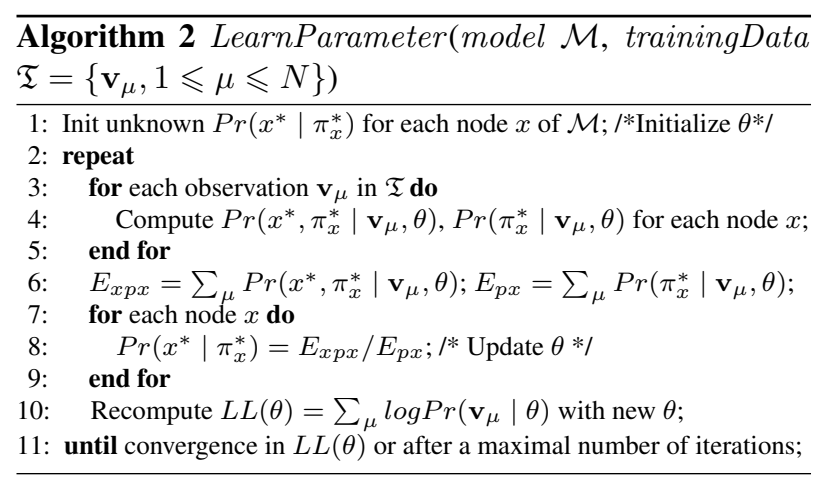

data hosting service may be initialized as in the service advertisement. Lines $3-6$ implement the Expectation step of the EM algorithm, where the expected counts $E_{x p x}$ and $E_{p x}$ of two joint events $\left(x^{*}, \pi_{x}^{*}\right)$ and $\pi_{x}^{*}$ are computed, given each observation $\mathbf{v}_{\mu}$ in collected reports and current parameter $\theta$. Any exact or approximate probabilistic inference algorithm can be used to compute the posterior probabilities $\operatorname{Pr}\left(\pi_{x}^{*} \mid \mathbf{v}_{\mu}, \theta\right)$ and $\operatorname{Pr}\left(x^{*}, \pi_{x}^{*} \mid \mathbf{v}_{\mu}, \theta\right)$. The Junction Tree Algorithm (JTA) [10], is a good candidate as it produces exact results, works for all QoS generative models, and is still computationally scalable as shown in our later analysis.

The Maximization step of the EM algorithm is implemented in lines $7-9$ of Algorithm 2, therein we update the model parameters $\operatorname{Pr}\left(x^{*} \mid \pi_{x}^{*}\right)$ such that they maximize the training data's likelihood, assuming that the expected counts computed in lines $3-6$ are correct. The two Expectation and Maximization steps are iterated till the convergence of the $\log$ likelihood $L L(\theta)$ of the training data set $\mathfrak{T}$, which gives us an approximation of unknown CPT entries $\operatorname{Pr}\left(x^{*} \mid \pi_{x}^{*}\right)$ of the QoS generative model.

The set of observable variables in the QoS generative model may be changed after a user runs Algorithm 2, uses the service, and updates statistics of some quality attributes $q_{i}$ with his own experience. Thus the learning of the model parameters is a reinforcement process with increasing accuracy over time, given availability of more training data to estimate a fewer number of parameters.

We do not consider possible optimization techniques, e.g., adjust the weight of each sample $\mathbf{v}_{\mu}$ according its recency, to accelerate the learning convergence. The use of other parameter learning algorithms, e.g., a Bayesian learning method, is also possible. These issues are subject to future work and thus beyond the scope of this paper.

Service quality estimation: Our goal is to compute the joint probability $\operatorname{Pr}\left(\mathcal{D}^{*} \mid \Phi^{*}\right)$ that the service offers a set $\mathcal{D} \subseteq \mathcal{Q}$ of quality attributes at a desired level $\mathcal{D}^{*}=\left\{q^{*}, q \in\right.$ $\mathcal{D}\}$ and under a client context $\Phi^{*}=\left\{e^{*} \mid e \in \Phi \subseteq \mathcal{E}\right\}$. This probability implies whether a service performs better than another in terms of the quality features $\mathcal{D}^{*}$ under environmental setting $\Phi^{*}$. Thus, the result can be used for 
ranking and selection of appropriate services among functionally equivalent ones given their expected quality levels. The trustworthiness of an untrusted feedback source $j$, i.e., $\operatorname{Pr}\left(b_{j}\right)$, is also of our interests, as this helps to select more reliable sources for future estimation, especially it is costly to obtain information from such feedback sources.

Given a generative QoS model with a known parameter $\theta$, the computation of the above probabilities using probabilistic inference algorithms is straightforward [10] as follows. Values of contextual variables in $\Phi$ are defined according to setting of the user, then a probabilistic inference algorithm, such as JTA [10] is run on the model to compute the probability $\operatorname{Pr}\left(\mathcal{D}^{*} \mid \Phi^{*}\right)$. Regarding the trustworthiness of feedback sources, all probabilities $\operatorname{Pr}\left(b_{j}\right)$ are already computed during the parameter learning step.

For example, suppose that we want to estimate the probability that the service with the QoS generative model in Fig. 2(c) provides data hosting service with download speed level $D=$ high. Also assume that the user wants a free service. The probability $\operatorname{Pr}(D=$ high $\mid P=$ free $)$ can be done automatically with available probabilistic inference algorithm, given a QoS generative model with known CPT entries. Due to space limitation, we refer readers to [10] for a comprehensive tutorial on possible inference algorithms. The most important aspect is if we know conditional probability entries of a QoS generative model, the whole above inference procedure can be done fully automatically with acceptable computational cost (Section 5).

\section{Analysis of the approach}

We provide in this section some theoretical results showing possibilities of our approach. These results are for the candidate algorithms in this paper and generally suitable to any service domain. Many optimizations, however, can be used to increase their effectiveness and efficiency.

We will estimate the most significant computational cost in our framework, which is the parameter learning and probabilistic inferences on the QoS generative model. Consider a worst case scenario where each of $m$ quality attributes has $t$ contextual factors as parent nodes. There are $n$ feedback sources being used, none of which are trusted. Suppose that every node has a $k$-ary state space. In a specific domain and for a certain service, $t, m$, and $k$ are fixed values, known to the service user, and typically much smaller than $n$. The Bayesian network of such a generative model has a total of $t+m+n+m n=O(n)$ nodes. The number of unknown CPT entries $\operatorname{Pr}\left(x^{*} \mid \pi_{x}^{*}\right)$ in the parameter $\theta$ is at $\operatorname{most} n_{\theta}=(k-1) t+(k-1) k^{t} m+(k-1) n+(k-1) k^{2} m n=$ $(k-1)\left(k^{2} m n+n+k^{t} m+t\right)=O(n)$. The term $k-1$ is due to the normalization constraints as each variable has a $k$-ary state space.

Assume that a source $j$ sends $N$ reports on some quality attributes. In fact, $N$ approximates the number of mea- surement epochs, or the number of samples obtained from Algorithm 1. The functions selectRatingsInEpoch and findReportedNode can be implemented with computational cost $O(1)$, e.g., with hash-based storage techniques. Since the cost of three loops in lines 1,3,7 of Algorithm 1 are respectively $O(N), O(n)$, and $O(t+m)$, the computational cost of Algorithm 1 is $O(N n(t+m))=O(N n)$. Even better, this step is usually done off-line. The computation that needs to be done on-demand is the estimate of $\operatorname{Pr}\left(\mathcal{D}^{*} \mid \Phi^{*}\right)$, which has a cost of $O(n)$ (Proposition 1).

Proposition 1. (see [13] for proof) The computational cost of one probabilistic inference on the worst-case QoS generative model using the Junction Tree Algorithm [10] is $O(n)$.

From Proposition 2, the cost of one EM iteration is $O\left(N n^{2}\right)$. Given the fact that in practice the EM algorithm converges fast, and we consider a limited number of feedback sources, Algorithm 2 is scalable in terms of computational cost with respects to the training data size $N$.

Proposition 2. (see [13] for proof) The computational cost of one EM iteration of Algorithm 2 is $O\left(\mathrm{Nn}^{2}\right)$.

Learning errors and sample complexity: the sample complexity to learn the parameter of a fixed-structure Bayesian network, or the required number of samples $N$ to learn $\theta$ with optimal error, is well-known $[11,14,15]$. In fact, the required number of samples in our framework is exponential in terms of the in-degree bound of nodes and grows less than linearly with the network size $[14,15]$. For the QoS generative model in our worst-case scenario with a bound of node's in-degree of $O(t+m)$ and network size of $O(n)$, the sample complexity is $N=O\left(n 2^{t+m}\right)$. This complexity implies that our approach is feasible for estimating QoS of services with a moderate number $t+m$ of quality attributes and contextual factors.

We implemented the proposed algorithms with the BNT toolbox ${ }^{6}$ and performed various experiments on the example data hosting service in our previous work [8]. Even with a high fraction (more than a half) of missing values from reports of several biased feedback sources, the quality of the estimation is relatively good. A more general implementation of the framework using $\mathrm{Netica}^{7}$ is available as a component of our reputation simulation framework ${ }^{8}$. However, a thorough experimentation of our framework with different services across service domains requires a lot of efforts and thus is subject to future work.

\section{Related work}

Many work propose to measure service quality using dedicated monitoring services or QoS brokers, namely [2,

\footnotetext{
${ }^{6} \mathrm{http}: / /$ bnt.sourceforge.net/

${ }^{7}$ http://www.norsys.com/netica.html

${ }^{8} \mathrm{http}: / /$ sirpeople.epfl.ch/lhvu/download/repsim/
} 
16-18]. Quality ratings from these sources are reliable yet expensive to obtain and maybe inscalable in terms of costs. Some work [2] is only applicable to measuring networkrelated performance metrics of Web services, e.g., responsetime, availability, but not for online services in general.

Many other approaches use user feedback to estimate quality and reputation of a (Web) service, namely [7, 1921]. However, they either assume full reliability of ratings or consider independent quality properties of a service. Also, most work are based on ad hoc heuristics that are not theoretically sound and the notions of context are usually not included.

Approaches most related to our work are in trust management literature $[4,5]$, which we do not survey due to space limitation. The work in this paper is based on our previous work on Bayesian modeling and learning of peer's quality [8]. In [22] the authors propose modeling e-market services as a Bayesian network and use a Bayesian learning approach to estimate distribution of the model parameters. [23] uses a simple Bayesian network to learn the trustworthiness of a party by the EM algorithm. These approaches do not exploit domain knowledge on service structure and presence of trusted parties to reduce the cost of probabilistic learning and inference on the models. As a side-effect, our framework, though independently developed, appears to subsume these specific approaches.

\section{CONCLUSION}

We have presented an overall framework for the probabilistic modeling and estimation of quality of online services, using feedback from both trusted and untrusted users on different quality attributes of the services. We present methods to exploit available knowledge in a service domain to build a probabilistic graphical model that generates ratings on a service's quality. Candidate algorithms for the model parameter learning and service quality estimations have been proposed and analyzed in details in the paper. These theoretical analysis and preliminary experiments show that the framework has a reasonable computational cost and is effective in elimination of possibly biased information in favor of or against certain service providers and thus gives an accurate picture of service quality for the selection and ranking of online services.

\section{References}

[1] J. O'Sullivan, D. Edmond, and A. H. M. ter Hofstede, "Formal description of non-functional service properties," Technical Report, Queensland Univ. of Tech., Australia, 2005.

[2] L. Fei, Y. Fangchun, S. Kai, and S. Sen, "A policy-driven distributed framework for monitoring quality of web services," ICWS'08, pp. 708-715, 2008.

[3] S. C. Wong, V. Tan, W. Fang, S. Miles, and L. Moreau, "Grimoires: Grid registry with metadata oriented interface: Ro- bustness, efficiency, security," IEEE Dist. Sys. Online, vol. 6, 2005.

[4] A. Jøsang, R. Ismail, and C. Boyd, "A survey of trust and reputation systems for online service provision," Decis. Support Syst., 43(2): 618-644, 2007.

[5] Y. Wang and K.-J. Lin, "Reputation-oriented trustworthy computing in e-commerce environments," IEEE Internet Computing, 12(4): 55-59, 2008.

[6] L. Shao, J. Zhang, Y. Wei, J. Zhao, B. Xie, and H. Mei, "Personalized qos prediction forweb services via collaborative filtering," ICWS'07, vol. 0, pp. 439-446, 2007.

[7] Z. Luo, K. Qian, D. Cai, and J. S. Li, "QoS driven web services assessment and selection," Int. J. Services Operations and Informatics, 1(1-2), 2006.

[8] L.-H. Vu and K. Aberer, "A probabilistic framework for decentralized management of trust and quality," CIA'07, 2007.

[9] D. Bianculli, W. Binder, L. Drago, and C. Ghezzi, "Transparent reputation management for composite web services," ICWS'08, pp. 621-628, 2008.

[10] C. Huang and A. Darwiche, "Inference in belief networks: A procedural guide," International Journal of Approximate Reasoning, 15(3): 225-263, 1996.

[11] W. Buntine, "A guide to the literature on learning probabilistic networks from data," IEEE Transactions on Knowledge and Data Engineering, vol. 8, no. 2, pp. 195-210, 1996.

[12] R. Neal and G. Hinton, "A view of the EM algorithm that justifies incremental, sparse, and other variants," in Learning in Graphical Models (M. I. Jordan, ed.), Kluwer, 1998.

[13] L.-H. Vu and K. Aberer, "Probabilistic Estimation Quality Ratings of Online Services," EPFL Tech. Rep., available at http://infoscience.epfl.ch/search?recid=131188\&of=hd.

[14] S. Dasgupta, "The sample complexity of learning fixedstructure bayesian networks," Mach. Learn., 29(2-3): 165180, 1997.

[15] P. Wocjan, D. Janzing, and T. Beth, "Required sample size for learning sparse Bayesian networks with many variables," Arxiv preprint cs.LG/0204052, 2002.

[16] S. Ran, "A model for Web services discovery with QoS," SIGecom Exch., 4(1): 1-10, 2003.

[17] M. Ouzzani and A. Bouguettaya, "Efficient access to Web services," IEEE Internet Computing, pp. 34-44, 2004.

[18] C. Patel, K. Supekar, and Y. Lee, "A QoS oriented framework for adaptive management of web service based workflows.," DEXA'03, pp. 826-835, 2003.

[19] Y. Liu, A. Ngu, and L. Zheng, "QoS computation and policing in dynamic web service selection," $W W W^{\prime} 04,2004$.

[20] E. M. Maximilien and M. P. Singh, "Agent-based trust model involving multiple qualities," AAMAS'05, 2005.

[21] L. Mei, W. Chan, and T. Tse, "An adaptive service selection approach to service composition," in ICWS'08, 2008.

[22] K. Regan, P. Poupart, and R. Cohen, "Bayesian reputation modeling in e-marketplaces sensitive to subjectivity, deception and change.," AAAI'06, 2006.

[23] J. Y.-J. Hsu, K.-J. Lin, T.-H. Chang, C.-J. Ho, H.-S. Huang, and W.-R. Jih, "Parameter learning of personalized trust models in broker-based distributed trust management," Information Systems Frontiers, 8(4), pp. 321-333, 2006. 